\title{
Time intermittency and spectral features of the geomagnetic field
}

\author{
Paola De Michelis $\left({ }^{1}\right)$ and Giuseppe Consolini $\left({ }^{2}\right)$ \\ ${ }^{(1)}$ Istituto Nazionale di Geofisica e Vulcanologia, Roma, Italy \\ $\left.{ }^{(}\right)$Istituto di Fisica dello Spazio Interplanetario - CNR, Roma, Italy
}

\begin{abstract}
In the field of geomagnetism a number of studies have been devoted to the investigation of turbulence and intermittency in the outer core fluid motions. Here, in order to obtain information on such phenomena we study the time spectral and self-similarity features of the main geomagnetic field fluctuations as measured on the Earth's surface. The existence of a power law spectrum, characterised by an exponent $\alpha \approx-11 / 3$, and an anomalous scaling of $q$-th order structure functions on time scales longer than 5 years, suggests the occurrence of intermittent turbulence rather than classical Kolmogorov turbulence in the fluid core motions. These results are briefly discussed in connection with the existence of a strong magnetic field and drift-wave turbulence.
\end{abstract}

Key words nonlinear processes in geophysics - geomagnetic field - Earth's core - fluid dynamics turbulence

\section{Introduction}

The geomagnetic field is sustained within Earth's outer core by dynamo action, which involves vigorous convective motions. It is widely thought that these fluid motions are driven by the Archimedean (buoyancy) force and are strongly affected by both the Coriolis and Lorentz forces. Moreover, it is commonly assumed that these motions are also fully turbulent. Direct observations of the fluid motions are unfortunately impossible and consequently the picture of the core turbulence is usually painted by theoretical reasoning. Actually, our understanding of the effect of turbulence in the core and of its influence on the diffusive processes is

Mailing address: Dr. Paola De Michelis, Istituto Nazionale di Geofisica e Vulcanologia, Via di Vigna Murata 605, 00143 Roma, Italy; e-mail: demichelis@ ingv.it rather limited. However, the study of this topic is essential because it could open new ways both to improve the reliability of geodynamo simulations and for a better understanding of all those phenomena which are connected with the dynamics of the fluid core motions.

Turbulent flows are generally characterized by «universal» scaling features in the so-called inertial range where fluctuations reach a stationary state due to the continuous flow of energy from large to small scales, or vice versa. This energy flow can be viewed in real space as an energy cascading process generated by the breakdown of eddies at different scale lengths or as a coalescence process in the case of an inverse energy cascade.

Although energy cascading is also expected to occur in many three-dimensional magnetohydrodynamic (MHD) systems, it is not clear if such a cascading process can occur in the Earth's core. Stevenson (1969) and Braginsky and Meytlis (1990) have considered the problem of the turbulence in the core, and attempted to qualitatively estimate the effects of turbulence. In particular, Braginsky and Meytlis (1990) suggested that the buoyancy instability, locally supercritical, should strongly affect the develop- 
ment of small-scale turbulence inhibiting a significant cascading of energy from the dominant and larger cells to the smaller ones. Thus, because of extreme conditions in the terrestrial core the core turbulence could be totally unlike classical Kolmogorov turbulence. Braginsky and Meytlis (1990) have also suggested that this small-scale turbulence should be highly anisotropic because of the strong influence of the Earth's rotation and magnetic field. They conclude their paper by suggesting that the study of data relative to the geomagnetic secular variation may be highly relevant to understand the dynamo processes. As a matter of fact, the characteristic time interval of the local turbulence lies in the range from 10 to 100 years (of the order of decades), and thus data about the decade geomagnetic secular variations may be relevant.

Here, taking this suggestion into account we attempt to investigate the nature of the Earth's fluid core turbulent motions using ground-based observations of the geomagnetic field. We would like to remark that our observational approach could be very useful in setting constraints on numerical models of the geodynamo. This paper is structured as follows. The mathematical details are largely confined to Section 2, in which we review some of the geomagnetic field equations and of the theory of turbulence and intermittency, while in Section 3 and 4 we present our analysis and conclusions, respectively.

\section{Analytical approach}

\subsection{The geomagnetic field equations}

The equation, which couples the main geomagnetic field and its secular variation to the fluid core motions, is the magnetic induction equation

$$
\partial_{t} \boldsymbol{B}=\nabla \times(\boldsymbol{V} \times \boldsymbol{B})+\eta \nabla^{2} \boldsymbol{B}
$$

where $\boldsymbol{V}$ is the velocity of the fluid, $\eta=(\mu \sigma)^{-1}$ is the magnetic diffusivity (with $\mu=4 \pi \times 10^{-7}$ $\mathrm{Hm}^{-1}$ the magnetic permeability and $\sigma=3 \times 10^{5}$ $\mathrm{S} / \mathrm{m}$ the electrical conductivity of the core fluid assumed constant) and $\boldsymbol{B}$ is the magnetic induction. The magnetic induction equation shows that the time rate of the change of the magnetic field (the secular variation) depends upon both magnetoadvection $\nabla \times(\boldsymbol{V} \times \boldsymbol{B})$ and diffusion $\eta \nabla^{2} \boldsymbol{B}$. If we associate time scales with these two different processes we find that, for the Earth's core, the typical time scale of advection is of the order of 60 years, while the time scale of diffusion is about $3 \cdot 10^{4}$ years (see Bloxham and Jackson, 1991). This difference between the time scales suggests omission of the diffusion term, at least over time scales short compared with the time scale of diffusion, and consideration of the reduced equation (Roberts and Scott, 1965)

$$
\partial_{t} \boldsymbol{B}=\nabla \times(\boldsymbol{V} \times \boldsymbol{B})=(\boldsymbol{B} \cdot \nabla) \boldsymbol{V} .
$$

This hypothesis, which is called the «frozenflux» hypothesis, is valid for time scales $T$ in the range $10<T<100$ years (Backus et al., 1996). For longer time scales the diffusion of the magnetic field throughout the fluid core is instead observable even if, as Bloxham et al. (1989) showed, the frozen flux condition is still valid and gives a good representation of core motions on a broad scale.

If we assume that the geomagnetic field $(\boldsymbol{B})$ and the fluid velocity $(\boldsymbol{V})$ can be decomposed according to their large-scale (macroscale) and small-scale (microscale) parts the former being the average over turbulence (dented by the subscript 0) and the latter being the small-scale fluctuating part

$$
\begin{aligned}
& \boldsymbol{B}=\boldsymbol{B}_{0}+\boldsymbol{b} \\
& \boldsymbol{V}=\boldsymbol{V}_{0}+\boldsymbol{v} .
\end{aligned}
$$

From the induction equation we obtain the equation for the turbulent variables (see Braginsky and Meytlis, 1990)

$$
\begin{gathered}
d_{t} \boldsymbol{b}=\left(\boldsymbol{B}_{0} \cdot \nabla\right) \boldsymbol{v}+\boldsymbol{L}^{b}+\boldsymbol{M}^{\boldsymbol{b}} \\
\boldsymbol{L}^{b}=(\boldsymbol{b} \cdot \nabla) \boldsymbol{V}_{0}-(\boldsymbol{v} \cdot \nabla) \boldsymbol{B}_{0} \\
\boldsymbol{M}^{b}=\nabla \times(\boldsymbol{v} \times \boldsymbol{b}-\langle\boldsymbol{v} \times \boldsymbol{b}\rangle) .
\end{gathered}
$$

Here $d_{t}=\partial_{t}+\boldsymbol{V} \cdot \nabla$. The term $\boldsymbol{L}^{b}$ is small because it is proportional to the gradients of the basic (averaged) quantities which change over a characteristic length that is much greater than the characteristic length over which the turbulent variables change. Consequently, the term $\boldsymbol{L}^{b}$ can be neglected. It is also necessary to stress that, 
though the role of the local turbulence in some internal processes is crucial, its influence on ohmic diffusion of the basic field and on the $\alpha$ effect is negligible. In particular, Braginsky and Meytlis (1990) have shown that both the coefficient of the $\alpha$-effect and the $\beta$-effect are small in the local turbulence and this makes both these nonlinear effects negligible. As a matter of fact, the $\alpha$-effect is most active at intermediate to large scales, and not at small scales, as classically assumed. This means that, in first approximation, the term $\boldsymbol{M}^{b}$ can be neglected too. Transforming to a moving coordinate system, we can set $\boldsymbol{V}_{0}=0$ and consequently, eq. (2.4) becomes

$$
\partial_{t} \boldsymbol{b}=\left(\boldsymbol{B}_{0} \cdot \nabla\right) \boldsymbol{v}=\nabla \times\left(\boldsymbol{v} \times \boldsymbol{B}_{0}\right) .
$$

In terms of Fourier components, eq. (2.7) reduces approximately to

$$
-i \omega \boldsymbol{b}_{k, \omega} \approx i \boldsymbol{k} \times\left(\boldsymbol{v}_{k, \omega} \times \boldsymbol{B}_{0}\right)
$$

where $\omega$ is the angular frequency and $\boldsymbol{k}$ is the wave number. Therefore, in scalar form we obtain

$$
\omega b_{k, \omega} \approx k B_{0} v_{\omega, k} .
$$

Expression (2.9) relates the temporal spectral features of the magnetic field to the spatial spectral features of the velocity fields in the limits imposed by the frozen-field hypothesis.

\subsection{A brief overview of turbulence}

The fundamental dynamical equation, describing the emergence of turbulence, is the Navier-Stokes equation, and the parameter, which describes the physical state of the fluid, is the so-called Reynolds number $R=L V / v$, where $L, V$ and $V$ are a characteristic length scale, the velocity of the flow and the kinematic viscosity, respectively. This number is a nondimensional measure of the nonlinearity effects in the Navier-Stokes equation. If the Reynolds number is not too large $(R<1)$, the fluid flow is laminar in the sense that it displays regular and predictable variations in both space and time. When the Reynolds number increases
$(R>1)$, the fluid flow undergoes a sequence of instabilities until, at some large enough value of the Reynolds number $(R>>1)$, it becomes fully turbulent and the various symmetries permitted by the Navier-Stokes equations are broken.

Kolmogorov's theory (K41) (Kolmogorov, 1962; Frisch, 1995) gives a description of the main mechanism acting in an incompressible fluid at high Reynolds number. According to Richardson's scenario, in a turbulent flow there is a cascade transfer of energy toward small scales where dissipation is due to molecular friction. The energy cascade is hierarchical, i.e. a disturbance on a certain scale receives its energy from a larger-scale disturbance and transfers it to smaller-scale disturbances. The presence of a range of length scales, where inertial forces are dominant and where viscous effects as well as the external forcing can be neglected, suggests the existence of universal scaling laws. As shown by Kolmogorov (1962), the main feature of a turbulent fluid flow is the existence of a statistical relation between the velocity differences $\delta v_{r}=|\boldsymbol{v}(\boldsymbol{x}+\boldsymbol{r})-\boldsymbol{v}(\boldsymbol{x})|$ and the energy transfer rate $\varepsilon_{r}$, at the scale $r=|\boldsymbol{r}|$

$$
\delta v_{r} \approx\left(\varepsilon_{r} r\right)^{1 / 3}
$$

which is generally known as Kolmogorov's refined similarity hypothesis. In terms of the energy spectrum $(E)$ in the inertial range this corresponds to the even more famous scaling law

$$
E(k) \propto k^{-5 / 3} \quad(k \text { being the wave-number) }
$$

The same picture can be extended to magnetohydrodynamic (MHD) turbulence, though the analogous statistical relations between the velocity differences and the energy transfer rate must take into account the Alfvén decorrelation effect (Kraichnan, 1965). As a consequence of the Alfvén decorrelation effect Kolmogorov's refined similarity hypothesis has to be replaced by

$$
\delta v_{r} \approx\left(c_{A} \varepsilon_{r}\right)^{1 / 4} r^{1 / 4}
$$

where $c_{A}=B /(4 \pi \rho)^{1 / 2}$ is the Alfvén velocity (in a medium of density $\rho$ and average magnetic field $B$ ) associated with the largest scale. Owing to 
this revised refined hypothesis in the case of MHD turbulence (Kraichnan, 1965), the famous $-5 / 3$ energy spectrum reduces to

$$
E(k) \propto k^{-3 / 2} .
$$

The aforementioned description of fluid and/or MHD turbulence is valid in the case of non-rotating systems and in the absence of a strong applied magnetic field. In fact, recent experimental results and numerical simulations have clearly shown that an intense magnetic field and/or rotation can strongly affect the spectral features of turbulent fluctuations in the inertial regime. Some recent experiments (see Branover et al., 1999) revealed that an increasing external magnetic field may change the velocity spectral features. For very intense magnetic fields, when the ratio between the Hartman number $(H a=B L \sqrt{\sigma / \rho \nu}$, where $B$ is the intensity of the magnetic field, $L$ is a characteristic length, $\sigma$ is the conductivity, $\rho$ is the fluid density, and $v$ is the fluid kinematic viscosity) and the Reynolds number $R$ exceeds some critical thresholds $\left(\theta_{i}\right)$, the velocity spectrum reduces to

$$
E(k) \propto k^{-5 / 3} \underset{\theta_{1}<H a / R<\theta_{2}}{\longrightarrow} k^{-7 / 3} \underset{H a / R>\theta_{2}}{\longrightarrow} k^{-11 / 3}
$$

and turbulence becomes anisotropic and strongly intermittent. A steeper energy spectrum is a consequence of the fact that the existence of an intense magnetic field can inhibit the formation of turbulent structures at small scales, thus reducing the energy transfer towards smaller scales.

A different framework for the development of turbulent regimes is due to drift-waves (generally known as Rossby waves) in quasi-geostrophic flows. In such a case the equation governing the evolution of flows on a rotating sphere is the well-known Charney equation (del-Castillo-Negrete, 2000)

$$
\begin{gathered}
{\left[\partial_{t}+(\boldsymbol{z} \times \nabla \psi) \cdot \nabla\right] q=0} \\
q=\nabla^{2} \psi-k_{r}^{2} \psi+\beta y
\end{gathered}
$$

where $z$ is the radial outward unit vector on the sphere, $\psi$ the streamfunction, $k_{r}$ the Rossby deformation wave number, and $\beta$ the Coriolis force in the quasi-geostrophic $\beta$-plane approximation. Equation (2.15) is formally equivalent to the Hasegawa-Mima equation, which is an important paradigm for the description of driftwave turbulence in magnetically confined plasmas. Recent studies (Ottaviani and Krommes, 1992; Kukharkin et al., 1995) of the drivendamped Hasegawa-Mima equation revealed the existence of different scaling regions with respect to a characteristic spatial scale $L$ in the case of a strong-turbulent regime. In more detail, for an inverse cascade process one obtains

$$
E(k) \propto\left\{\begin{array}{l}
k^{-11 / 3} \Rightarrow k<L \\
k^{-5 / 3} \Rightarrow k>>L
\end{array}\right.
$$

while for a direct cascade the Hasegawa-Mima equation leads to

$$
E(k) \propto\left\{\begin{array}{l}
k^{-5} \Rightarrow k<L \\
k^{-3} \Rightarrow k>>L
\end{array} .\right.
$$

Again different scaling features are obtained depending on the kind of turbulence and on the different dynamical situations.

\subsection{A brief overview of intermittency}

An exact definition of intermittency is somewhat difficult to provide mainly because the term assumes different meanings in different physical frameworks. However, space-time intermittency seems to be a common feature of fluctuations, characterizing most «weakly confined» or «extended» systems in nature, for which the fluctuations are not uniformly distributed in space and/or time. For example, in the case of MHD or fluid turbulence, the term intermittency is generally used to indicate a non-uniform distribution of energy in space at a given length scale, and is associated with a multifractal structure (i.e. a local dependence of the self-similarity features) of the energy cascade in the inertial range. The approach to intermittency has been applied for a long time. Although some possible mechanisms have been proposed to explain intermittency in many physical frameworks, it seems that a comprehensive knowledge of this universal phenomenon has not been completely reached, requiring 
a detailed understanding of coupling among micro-, meso- and macroscale fluctuations.

In turbulent fluid flows intermittency is usually investigated by looking at the self-similarity features of measurable quantities such as the $q$-th order structure velocity functions $S_{r}^{(q)}$, defined as the spatial averages of $\delta v_{r}^{q}=|v(x+r)-v(x)|^{q}$. In detail, one can define a hierarchy of scaling exponents $\xi_{q}$ through the following scaling relation:

$$
S_{r}^{(q)}=\left\langle\left|\delta v_{r}\right|^{q}\right\rangle \approx r^{\xi q}
$$

where, in the absence of intermittency effects, the scaling exponents $\xi_{q}$ are expected to scale linearly as a function of the moment order $q$. Abundant experimental and numerical evidence (Frisch, 1995) suggests that the exponents $\xi_{q}$ are different from their classical values, and do not scale linearly as a function of moment order $q$. These departures are the signature of intermittency, i.e. of a non-homogeneous fragmentation of eddies, which leads to a non-homogeneous distribution of energy at the smaller scales. In the case of «standard» fully developed fluid turbulence, starting from Kolmogorov's «refined similarity hypothesis» (Kolmogorov, 1962) intermittency effects can be included through a renormalization of the scaling exponents $\xi_{q}$ as follows:

$$
\xi_{q}=\frac{q}{m}+\tau_{q}
$$

where $m=3$ or 4 for fluid or MHD turbulence, respectively. Several models have been proposed to describe intermittency by deriving an expression for $\xi_{q}$ (Benzi et al., 1984; Meneveau and Sreenivasan, 1987; Carbone, 1993, 1994; Grauer et al., 1994; She and Leveque, 1994; Politano and Pouquet, 1995). Taking into account the effects of intermittency, each of these models generally predicts $\tau_{q}>0$ for $q / m<1$, and $\tau_{q}<0$ for $q / m>1$; i.e. $\xi_{q}$ is a convex function of $q$.

\section{Data set and analysis}

In order to study the nature and/or the occurrence of intermittency and turbulence in the geomagnetic time series and obtain information on turbulence in the fluid core, ground magnetic observatory data were used. In particular, in this paper the annual mean values of the three geomagnetic field components $(X, Y$ and $Z)$ relative to 18 observatories, mostly in the Northern Hemisphere, formed the data set. The observatories were selected in order to have more than 94 years of annual mean values available. Data come from both the National Geophysical Data Center, Boulder (CO), and the «Summary of annual means at world magnetic observatories» (Golovkov et al., 1983). Figure 1 shows the dis-

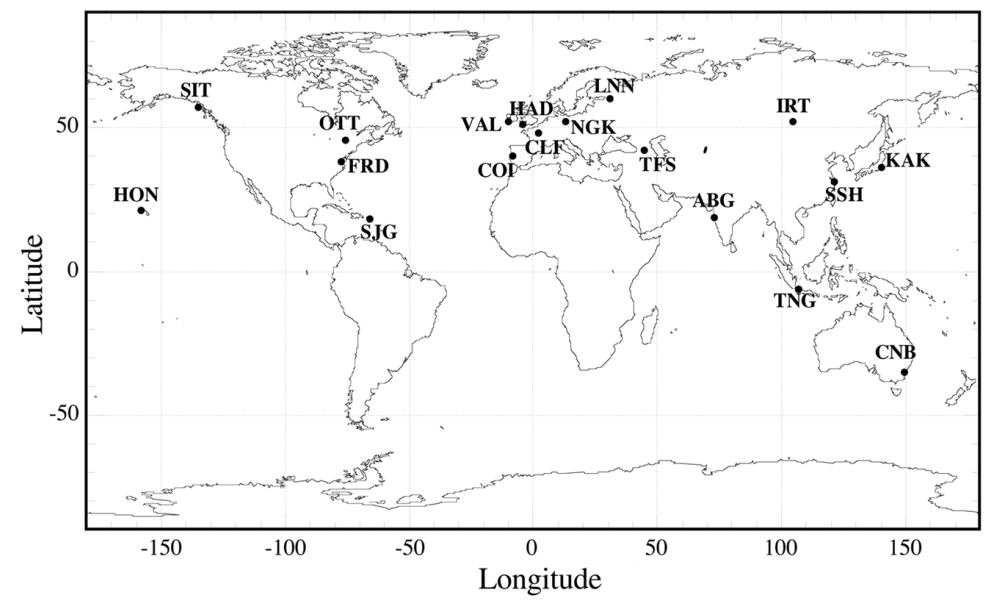

Fig. 1. World-map of the location of the geomagnetic observatories used in this work. 
tribution of the observatories used in this work. Since some of the observatories were re-located during the time interval considered, when this happened, data were corrected shifting their absolute level appropriately adjusting the annual means to the level appropriate to the latest site. Data gaps were linearly interpolated only when the number of missing points is equal to one, otherwise the time series was rejected.

First of all, the spectral features of the geomagnetic field have been investigated to keep information on the dynamical state of the fluid core motions. In order to do this, from eq. (2.9) we obtained the following relation for the energy spectra:

$$
E_{B}(\omega)=\left(\frac{k}{\omega}\right)^{2} B_{0}^{2} E_{v}(k)
$$

where $E_{B}(\omega)$ and $E_{v}(k)$ are the Power Spectral Densities (PSD) for magnetic and velocity fluctuations, respectively. Figure 2 shows the average PSD $S(f)$ defined as follows:

$$
S(f)=\left\langle\left(\sum_{i=\{X, Y, Z\}} \delta B_{j i}^{2}(f)\right)\right\rangle
$$

where $\delta B_{j i}^{2}(f)$ is the squared Fourier amplitude of the $i$-th component at the $j$-th observatory, and $f=\omega / 2 \pi$. The average $S(f)$ was evaluated after normalizing the result of each observatory at the lowest significant frequency $f^{*}=0.03 \mathrm{yr}^{-1}$. Due to the existence of long-term trends in the time series considered, we used a raised cosine (Hanning) window before performing the spectral analysis. Frequencies below $f^{*}$ were not considered since they are affected by the windowing procedure.

The PSD is characterized by the existence of two different scaling regimes respectively at frequencies below and above a certain crossover frequency $f_{C}$. In detail, for frequencies below $f_{C} \approx 0.2 \mathrm{yr}^{-1}$ the power spectrum follows a power-law with a spectral exponent $\alpha$ close to $-11 / 3(\alpha=-3.73 \pm 0.03)$ while above the crossover frequency $f_{C}$ the PSD seems to agree with a power-law, characterized by a spectral exponent $\alpha$ close to $-5 / 3$. We remark that our PSD, on time scales longer than 10 years, agrees with a previous analysis by Filloux (1980). The composite spectrum by Filloux (1980) has, indeed, a $1 / f^{4}$ dependence in

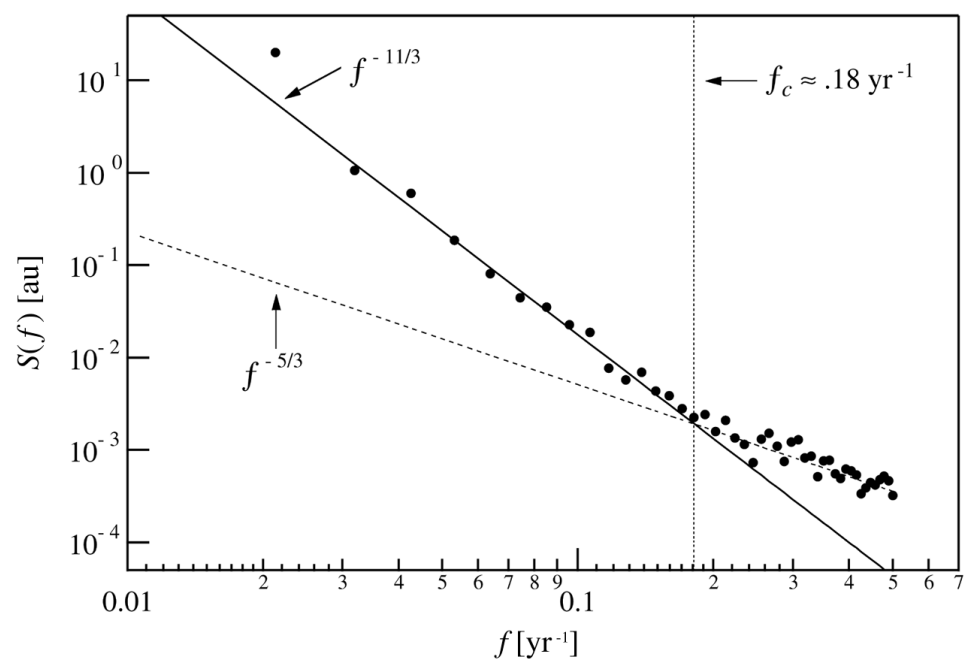

Fig. 2. The average PSD of the geomagnetic field fluctuations evaluated as described in the text. The solid and dashed lines refer to non-linear best fit using a power law. Spectral slopes are approximately $-11 / 3$ and $-5 / 3$ for frequencies lower or higher than the crossover frequency $f_{\mathrm{c}} \approx 0.2 \mathrm{yr}^{-1}$. The vertical dotted line denotes to the crossover frequency. 
the range of time scales from $\sim 1$ to $\sim 100$ years tapering to a less steep spectrum on the lowfrequency side (time scales $>100$ years). Actually, the spatial and temporal spectral features of the geomagnetic field have been widely investigated in the past and an interesting schematic power spectrum of the geomagnetic field combining data from Filloux (1980), Barton $(1982,1983)$ and the suggestion of $F$. Lowes (in Barton 1982) at the longest period is reported in the paper by Courtillot and Le Mouël, (1988). This schematic power spectrum begins in the range of observatory records with a $1 / f^{4}$ slope and tapers to a $1 / f$ for the longest paleosecular variation records of lake sediments and marine cores while the central portion is more like $1 / f^{2}$. However, the aim of this work is to try to understand the origin of such a spectrum in connection with spatial features of the fluid-core velocity pattern.

Because of the great difference between the diffusion characteristic time and the advection time we may assume that any local fluctuation of the velocity field can be considered as frozen-in in the range of time scales here considered ( $\sim 1-100$ years). Thus, these local fluctuations are essentially advected with a constant velocity without being dispersed. In terms of spatial $(\lambda)$ and time $(\tau)$ scales this is equivalent to assuming a linear relationship between $\lambda$ and $\tau$, i.e. a fluctuation of scale $\lambda_{f}$ is advected in a time $\tau_{f}=\lambda_{f} / v_{a d}$. This is equivalent to assuming a linear relationship between $k$ and $\omega$. We emphasise that such a hypothesis of inferring spatial scales from temporal scales is generally assumed in fluid turbulence and is referred to as Taylor's hypothesis (Frish, 1995).

Thus, on the basis of eq. (3.1) we recover for the velocity field spectral features

$$
E_{v}(k) \propto k^{-11 / 3} .
$$

Such a steep spectral slope $(\alpha=-11 / 3)$ suggests that small-scale fluctuations are strongly inhibited and that long-range order as well as coherent structures could be present in the velocity pattern.

The existence of a steep spectrum could also involve the occurrence of intermittency in the fluid motions. Again, assuming the frozen- flux hypothesis to be valid, this intermittency should be evident in the geomagnetic field time fluctuations; i.e. geomagnetic field fluctuations should be characterized by time-intermittency. To investigate this behaviour and to show more clearly the existence of this intermittency, we analysed the geomagnetic field structure functions for different values of the order $q$ defined as follows:

$$
S^{(q)}(\tau)=\left\langle\left|B_{j i}(t+\tau)-B_{j i}(t)\right|^{q}\right\rangle
$$

where $B_{j i}$ is the $i$-th component of the $j$-th observatory, $\tau$ is a time lag, and $q$ covers in the interval $[0,4]$ with a step of 0.2 . Figure 3 displays the behaviour of the $q$-th order structure functions $S^{(q)}$ versus $t$ for a limited set of $q$ in the interval $[1,4]$. We limited our investigation up to the 4th order because of the paucity of the available statistics, the total amount of data being about $6 \cdot 10^{3}$ points. These structure functions have been obtained by averaging over the three magnetic field components and all selected observatories. Figure 3 shows that for each moment order a range of time scales can be found where the structure function can be fitted using a power law; i.e.

$$
S^{(q)} \propto \tau^{\xi_{q}}
$$

where $\xi_{q}$ is the scaling exponent at the moment order $q$. This range generally extends over time scales longer than $\tau \approx 5$ years up to $\tau \approx 50$ years.

Figure 4 shows the scaling exponents $\xi_{q}$ versus the moment order $q$. The scaling exponents seem to deviate from a linear behaviour meaning that the slope varies nonlinearly with increasing $q$. This result is consistent with a multifractal nature of the geomagnetic field fluctuations and therefore with the occurrence of intermittency, confirming previous results by the authors (De Michelis et al., 1998) and by Vörös and Gianibelli (1998). Experimental measurements and direct numerical simulations of three-dimensional turbulent flows have, in fact, clearly shown how the self-similar energy cascade occurs through the repeated fragmentation of the vorticity field and how this process generates elongated spatial structures (vorticity tubes) which form a multifractal set where dis- 


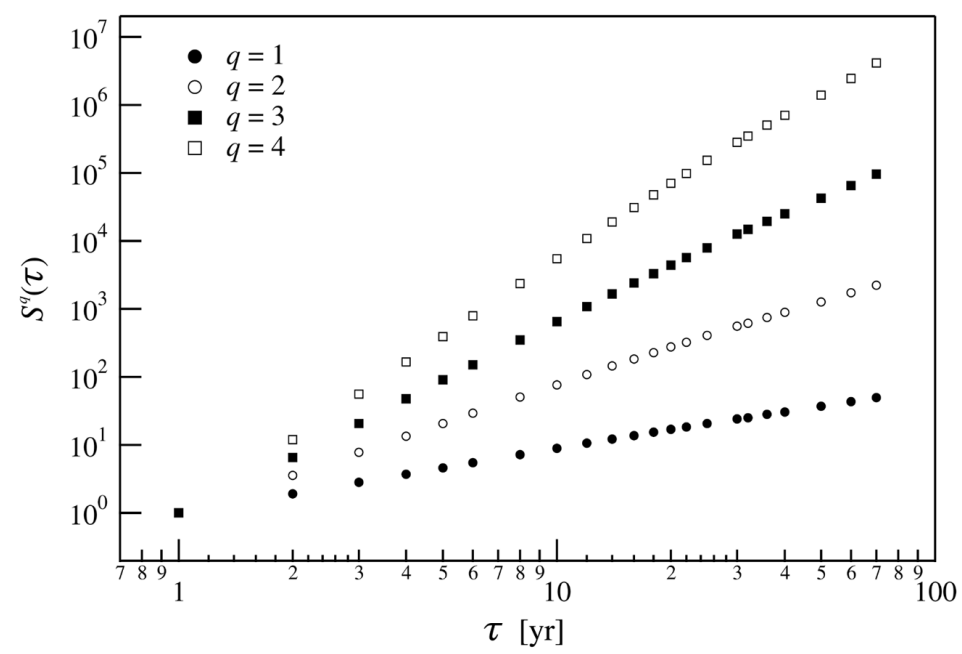

Fig. 3. The behaviour of the structure function $S^{q}(\tau)$ of the magnetic field fluctuations as a function of $\tau$ for a selected number of moment orders. Note the nearly linear trend in the double logarithmic plot meaning that geomagnetic field fluctuations are self-similar.

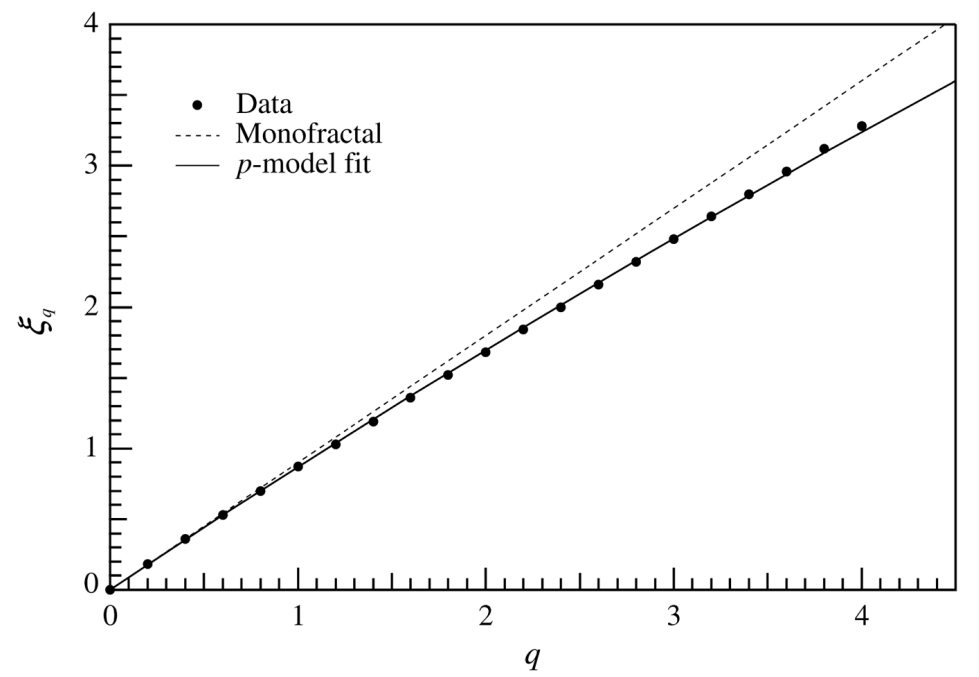

Fig. 4. The structure function scaling exponents $\xi_{q}$ versus the moment order $q$. The solid black line refers to a nonlinear best fit using the $p$-model. The resulting fragmentation parameter is approximately $p \approx 0.6$. The dashed line refers to a homogeneous fractal signal.

sipation eventually takes place. This contrasts with the K41 theory of homogeneous turbulence where a self-similar energy cascade was considered as a homogeneous not intermittent phenomenon. In the case of standard fully developed fluid turbulence, intermittency effects yield $\xi_{q}$ values slightly greater than $q / m$. In the last two decades, many different models have 
been proposed to describe intermittency by deriving an expression for $\xi_{q}$. Among these models that have been generally built up in the framework of hydrodynamic turbulence and successively extended to MHD turbulence, we note the $P$-model (Meneveau and Sreenivasan, 1987). The $P$-model, which is based on a twoscale Cantor set with equal partition intervals but unequal weights, conjectures that in the cascade process two «daughter» eddies are randomly generated from a «mother» eddy with a probability $p$ and $(1-p)$ (where $0 \leq p \leq 1)$ predicting

$$
\xi_{q}=1-\log _{2}\left[p^{q / m}+(1-p)^{q / m}\right] .
$$

We have used this model to take into account the intermittency effects and a very good agreement is found between the behaviour of the scaling exponents $\xi_{q}$ and the prediction of the $P$-model with a fragmentation ratio $p=(0.61 \pm$ $\pm 0.01)$.

\section{Summary and conclusions}

In the field of geomagnetism, many studies are devoted to understanding the nature of the physical features of the motions inside the Earth's core as well as to their relation with the maintenance of the main field. Although considerable progress has been made, much remains to be understood.

Here, as a contribution to the knowledge of the Earth's core processes, we have investigated the spectral and self-similarity features of the geomagnetic field recorded at 18 permanent observatories. We related the magnetic field spectrum to the core fluid velocity spectrum via the frozen flux approximation and Taylor hypothesis. Our findings suggest that above a certain critical scale $\lambda_{c}=k_{c}^{-1}$ (related to the characteristic frequency $f_{C} \approx 0.2 \mathrm{yr}^{-1}$ ) the spectrum drops off faster than one might expect for a Kolmogorov fluid and/or Kraichnan MHD turbulence. This result suggests that small-scale fluctuations are strongly inhibited and supports Braginsky and Meytlis' (1990) theoretical point of view of a non-traditional turbulence. Moreover, evidence of intermittency in the dynamics of the geomagnetic field has been found by analyzing the average scaling properties of the geomagnetic field fluctuations. In particular, the existence of an anomalous scaling of the structure function scaling exponents $\xi_{q}$ supports the occurrence of intermittency in the fluid core motions.

A possible origin of the steeper spectrum and of the observed time-intermittency could involve the presence of an intense core magnetic field, which would cause a rearrangement of the topological structure of the turbulent flow, modifying the velocity field spectral features. As already mentioned (Branover et al., 1999), there is experimental evidence that under the effect of an increasing external magnetic field the velocity spectrum evolves from Kolmogorov $-5 / 3$ exponent towards a steeper one in intense magnetic fields, and that the fluid motions should be strongly intermittent. In other words, the observed spectral slope could be consistent with an intermittent turbulent fluid dynamics in the outer core.

A different framework that could take account of the $k^{-11 / 3}$ spectrum, and that could also clarify the origin of the spectral crossover, might be the formation of coherent vortical structures in the fluid core in a drift-wave turbulent regime. In the outer fluid core, the smallscale fluctuations are restricted to the plane perpendicular to the magnetic field and that makes the turbulence essentially two-dimensional. If we take into account that the turbulence regime in the Earth's core could also be the result of drift-waves (Rossby waves), we may describe the problem of the turbulence dynamics of the fluid motions in the Earth's core using the Hasegawa-Mima (HM) equation. In the case of an inverse cascade this equation, which has a structure identical to that of the Charney equation, predicts two asymptotic inertial regimes for small or large wave numbers $k$ with respect to a characteristic scale $L=1 / k_{c}$ (see eq. (2.16)). Our results on the spectral features of the geomagnetic field show the existence of these two scaling regimes well in agreement with the solutions of Hasegawa-Mima equation. Thus we can expect that while the steep energy spectrum impedes the formation of small-scale coherent vortices simultaneously, as a consequence of 
the piling up of the energy at larger scales, there would be a promoting of the formation of longlived coherent vortices at large-scales.

We should, however, emphasise that the possible origin of the high frequency $-5 / 3$ spectrum could be different. For time scales shorter than 10 years the evaluation of the spectral features of the geomagnetic fluctuations is affected by two fundamental problems. First of all, even though we have used the magnetic field annual means the contamination due to external field sources remains as a major source of noise in this temporal range. Second, we must take into account that any magnetic signal with a period less than approximately 4 years, generated inside the core and crossing the core-mantle boundary, is effectively cut off from observation by the conducting lower mantle.

In summary, we have shown that it may be possible to obtain information on the outer core fluid motions using ground-based geomagnetic field measurements. Clearly, more work is necessary in order to clarify the proposed dynamical models, this will be the subject of future studies.

\section{REFERENCES}

Backus, G., Parker, R. and Constable, C. (1996): Foundations of Geomagnetism (Cambridge University Press), p. 249

Barton, C.E. (1982): Spectral analysis of palaeomagnetic time series and the geomagnetic spectrum, Phylos. Trans. R. Soc. London Ser. A, 306, 203-209.

BARTON, C.E. (1983): Analysis of paleomagnetic time series-technique and applications, Geophys. Surv., 5, 335-368.

Benzi, R., G. Paladin, G. Parisi and A. Vulpiani (1984): On the multifractal nature of fully developed turbulence and chaotic systems, J. Phys. A: Math. Gen., 17 (18), 3521-3531.

BLOXHAM, J. and A. JACKSON (1991): Fluid flow near the surface of the Earth's outer core, Rev. Geophys., 29, 97-120.

Bloxham, J., D. Gubbins and A. Jackson (1989): Geomagnetic secular variation, Philos. Trans. R. Soc. London, A329, 415-502.

BRAGINSKY, S.I. and V.P. MeytLIS (1990): Local turbulence in the Earth's core, Geophys. Astrophys. Fluid Dyn., 55, 71-87.

Branover, H., S.S. Moiseev, E. GolbraikH and A. EidelMAN (1999): Turbulence and Structures: Chaos, Fluctuations, and Helical Self-Organization in Nature and Laboratory (San Diego, Academic Press), pp. 270.
Carbone, V. (1993): Cascade model for intermittency in fully-developed magnetohydrodynamic turbulence, Phys. Rev. Lett., 71, 1546-1548.

CARbone, V. (1994): Scaling exponents of the velocity structure functions in the interplanetary medium, Ann. Geophysicae, 12, 585-590.

Courtillot, V. and J.L. LE MouËL (1988): Time variations of the earth's magnetic field. From daily to secular, Ann. Rev. Earth Planet. Sci., 16, 389-476.

De Michelis, P., G. Consolini and A. Meloni (1998): Sign singularity in the secular acceleration of the geomagnetic field, Phys. Rev. Lett., 81, 5023-5026.

Del Castillo-Negrete, D. (2000): Chaotic transport in zonal flows in analogous geophysical and plasma systems, Phys. Plasmas, 7, 1702-1711.

FillouX, J.H. (1980): Observation of very low frequency electromagnetic signals in the ocean, J. Geomagn. Geoelectr., 32 (supplement to n. 1), 1-12.

Frisch, U. (1995): Turbulence: the Legacy of A.N. Kolmogorov (Cambridge University Press), pp. 296.

Golovkov, V.P., G.I. Kolomitzzeva, L.P. Konyashenko and G.M. SemYonova (1983): The summary of annual mean values of magnetic elements at world magnetic observatories, Acad. Sci. URSS, XVI.

Grauer, R., J. Krug and C. MarLiani (1994): Scaling of high-order structure functions in magneto-hydrodynamic turbulence, Phys. Lett. A, 195, 335-338.

Kolmogorov, A.N. (1962): A refinement of previous hypothesis concerning the local structure of turbulence in a viscous incompressible fluid at high Reynolds number, J. Fluid Mech., 13, 82-85.

KRAICHNAN, R.H. (1965): Inertial range spectrum of hydromagnetic turbulence, Phys. Fluids, 8, 1385-1390.

KuKHARKIN, N., S.A. ORZAG and V. YAKHOT (1995): Quasicrystallization of vortices in drift-wave turbulence, Phys. Rev. Lett., 75, 2486-24888.

Meneveau, C. and K.R. SReEnivasan (1987): Simple multifractal cascade model for fully developed turbulence, Phys. Rev. Lett., 59, 1424-1427.

OTtAVIANI, M. and J.A. Krammes (1992): Weak- and strong-turbulence regimes of the forced Hasegawa-Mima equation, Phys. Rev. Lett., 69, 2923-2926.

Politano, H. and A. Pouguet (1995): Model of intermittency in magnetohydrodynamic turbulence, Phys. Rev. $E$, 52, 636-641.

RoberTs, P.H. and S. ScOTT, S. (1965): On the analysis of the secular variation, 1 . A hydromagnetic constraint: theory, J. Geomag. Geoelectr., 17, 137-151.

SHe, Z.-S. and E. LeVEQUE (1994): Universal scaling laws in fully developed turbulence, Phys Rev. Lett., 72, 336-339.

STEVENSON, D.J. (1969): Turbulent thermal convection in the presence of rotation and a magnetic field: a heuristic theory, Geophys. Astrophys. Fluid Dyn., 12, 139-169.

VöRÖs, Z. and J.C. GIANIBELLI (1998): On intermittent fluctuations of the earth's fluid core motions, Contrib. Geophys. Geod., 28, 277-285.

(received October 14, 2003; accepted March 7, 2004) 